\title{
Uma Proposta de Gestão de Projetos Para uma Universidade Pública
}

\section{A Project Management Proposal for a Public University}

\author{
Cícero Samuel Clemente Rodrigues ${ }^{1}$, Felipe Santana Furtado Soares ${ }^{2}$ \\ ${ }^{1}$ Diretoria de Tecnologia da Informação, Universidade Federal do Cariri, Juazeiro do Norte, Brasil \\ ${ }^{2}$ Mestrado Profissional em Engenharia de Software, CESAR School, Recife, Brasil \\ Correspondência: Cícero Samuel Clemente Rodrigues, Universidade Federal do Cariri, Endereço: R. Ten. \\ Raimundo Rocha, s/n, Cidade Universitária, CEP.: 63048-080 Juazeiro do Norte, Brasil. Tel: 5588 3221-9400. \\ e-mail: samuel.rodrigues@ufca.edu.br
}

Recebido: 5 de outubro de 2018 Aceito: 30 de novembro de 2018 Publicado: 31 de dezembro de 2018

DOI: http://dx.doi.org/10.21714/1679-18272018v16Ed.p264-278

\begin{abstract}
Resumo
Uma empresa de sucesso incorpora um fluxo de projetos para lidar com as mudanças que são fundamentais tanto em empresas públicas como privadas. Um levantamento do Tribunal de Contas da União (TCU) constatou através do índice de governança de tecnologia da informação (iGovTI) que $42 \%$ das instituições públicas não adotam práticas de gestão de projetos, potencializando o insucesso dos projetos de tecnologia da informação. No presente trabalho, utilizando a Universidade Federal do Cariri como campo do estudo, um processo simplificado de gestão de projetos foi modelado e formalmente implantado, juntamente com práticas e ferramentas para apoio. Para possibilitar um maior acompanhamento da comunidade acadêmica, foi criado um painel para monitoramento dos projetos. A adoção das práticas resultou no aumento significativo no nível de gerenciamento de projetos do iGovTI, possibilitou acompanhamento da comunidade para com os projetos de TI, e satisfação dos clientes envolvidos com processo e resultados dos projetos.
\end{abstract}

Palavras-chave: Gestão de Projetos, Gestão Pública, Projetos de TI.

\begin{abstract}
A successful company incorporates a stream of projects to deal with changes that are critical in both public and private companies. A survey by the Brazilian Tribunal de Contas da União (TCU) found through the Information Technology Governance Index (iGovTI) that $42 \%$ of public institutions do not adopt project management practices, boosting the failure of information technology projects. In the present study, using the Universidade Federal do Cariri as a field of study, a simplified project management process was modeled and formally implemented, along with practices and tools for support. In order to allow a greater follow-up of the academic community, a panel was created to monitor the projects. The adoption of the practices resulted in a significant increase in the level of project management of iGovTI, enabling the community to follow up on IT projects, and customer satisfaction involved with process and project results.
\end{abstract}

Keywords: Project Management, Public Management, IT Projects.

Esta obra está licenciada sob uma Licença Creative Commons Attribution 3.0.

\section{Introdução}

A administração pública vem buscando estratégias para uma maior governança, eficiência na execução, controle e consecução dos resultados previstos dos planos governamentais. (PISA; OLIVEIRA, 2014)

Os projetos são uma forma de alcançar metas e objetivos do plano estratégico organizacional, seja de forma direta ou indireta (PMI, 2017), entretanto, apesar do sucesso da abordagem de projetos na iniciativa privada, a administração pública possui características que dificultam a adoção dessas práticas (MACIEL; TEIXEIRA, 2012). Projetos públicos têm características especificas, como a natureza política, estão sujeitos a atenção da mídia e mais 
Uma Proposta de Gestão de Projetos Para uma Universidade Pública Revista Gestão.Org, v. 16, Edição Especial, 2018. p. 264-278

ISSN 1679-1827

http://www.revista.ufpe.br/gestaoorg

interessados, bem como demandam governança com foco em transparência (AZMAN; ABDUL-SAMAD; ISMAIL, 2013; CRAWFORD; HELM, 2009; KWAK; ANBARI, 2012).

É emergente a abordagem de temáticas a cerca de gestão de projetos no setor público uma vez que há poucas pesquisas específicas nesse campo (CLEMENTE; MARX; CARVALHO, 2017).

Do ponto de vista acadêmico, a maior parte dos trabalhos abordam métodos aplicados a projetos públicos são primordialmente em construção e infraestrutura, em geral, que geralmente incorporam a participação do setor privado (CLEMENTE; MARX; CARVALHO, 2017; EDER et al., 2012).

Considerando o ambiente de "multiversidade", conceito cunhado por Kerr (1982), o modelo decisório democrático das universidades é algo diferenciado dos demais órgão da administração pública, estruturado em órgãos de deliberação coletiva.

Segundo TCU 42\% instituições públicas não adotam processo de gerenciamento de projetos formal ou informal (BRASIL. TCU, 2014a). "Essa situação potencializa o risco de insucesso de projetos, sobretudo pela complexidade que, em regra, envolve o desenvolvimento e implantação de soluções de TI, prejudicando o alcance dos resultados esperados" (BRASIL. TCU, 2014a). Frente a isso, chega-se ao questionamento: como estruturar a gestão os projetos de TI, no contexto de uma universidade pública?

O presente estudo teve com objetivo, melhorar o acompanhamento de projetos de TI no contexto de uma universidade pública federal a partir da adaptação e implantação de práticas e ferramentas de gestão de projetos.

\section{Fundamentação}

\subsection{Projetos na Administração Pública}

Segundo o PMBoK, projeto é um esforço de natureza temporária empregado para atingir um objetivo, que geralmente se traduz em um produto, serviço ou resultado exclusivo, tendo seu início e termino bem definido (PMI, 2017). Segundo Cavalho Júnior (2012), as principais características de um projeto, as quais o distinguem das operações, são a temporariedade, exclusividade e a progressividade.

O PMBoK (PMI, 2017) afirma ainda, que o gerenciamento de projetos é a "aplicação de conhecimentos, habilidades, ferramentas e técnicas às atividades de projeto para atender aos seus requisitos".

Em projetos privados a principal meta é o lucro, sendo este lucro, fruto da relação entre produtor e consumidor, onde ao atender e necessidades desses consumidores, visa fundamentalmente obter o pagamento pelo consumo do bem produzido. O objetivo dos projetos nesse campo visa então, ou produzir o bem propriamente dito, ou apoiar o processo de produção desses bens, garantindo a rentabilidade e que esse processo seja autossustentável, garantindo a constante produção Cavalho Júnior (2012).

Já projetos no setor público sua meta é gerar o melhor resultado possível a população considerando que o público é o principal objetivo de sua existência, e não o lucro (CARVALHO JÚNIOR, 2012; MACIEL; TEIXEIRA, 2012).

Isso acaba construindo dificuldades diferenciadas (além das demais comuns à gestão de projetos), pois são políticos em sua essência, estão sujeitos a atenção da mídia e dos interessados em um formato mais transparente, possuem leis governamentais de determinam o que pode ser feito (órgãos e agentes públicos só podem agir no que é previsto em lei) e gestão sujeitos a pressão pública (CLEMENTE; MARX; CARVALHO, 2017).

\subsection{O guia PMBoK}

O Guia do Gerenciamento de Projetos (Guia PMBoK) tem como principal objetivo identificar conhecimento acerca de gestão de projetos amplamente conhecido como boa prática, considerando que "amplamente conhecido" significa que são aplicáveis à maioria dos projetos e que "Boa prática" significa que existe um consenso que determina que esses conhecimentos podem aumentar as chances de sucesso dos projetos (PMI, 2017).

Considerando que um processo é um conjunto de atividades inter-relacionadas, caracterizada por suas entradas, técnicas e ferramentas, objetivando criar um serviço ou produto, o PMBoK estrutura os processos de gerenciamento de projetos em cinco grupos de processos: Iniciação, planejamento, execução, monitoramento e controle, e encerramento (FOGGETTI, 2014; PMI, 2017; VARGAS, 2005), e são descritos abaixo: 
- Grupo de processos de iniciação: processos executados para definir um novo projeto pela obtenção da autorização para inicia-lo;

- Grupo de processos de planejamento: processos necessários para definir o escopo do projeto, a linha base do projeto e refinar seus objetivos;

- Grupo de processos de execução: processos que realizam o trabalho definido no plano, de forma a implementar as especificações do projeto.

- Grupo de processos de monitoramento e controle: processos necessários para acompanhar e controlar o progresso e o desempenho do projeto, além de identificar e controlar as mudanças no projeto.

- Grupo de processos de encerramento: processos executados para formalizar o encerramento do projeto.

Esses processos que compõem esses grupos são distribuídos em 9 áreas de conhecimento em gerenciamento de projetos, sendo elas; escopo, cronograma, custos, recursos, qualidade, comunicações, riscos e integração.

\subsection{O sucesso dos Projetos}

O conceito que determina se um projeto tem sucesso ou não é definido de forma ambígua pela literatura (BACCARINI, 1999; GRAY, 2001; KARLSEN, ANDERSEN; BIRKELY; ØDEGÅRD, 2005). O sucesso de um projeto vem tradicionalmente sendo representado na forma de um triângulo entre custo, tempo e objetivos de qualidade, de forma que, se esse projeto termina no tempo, dentro do orçamento e de acordo com as especificação, é determinado que foi concluído com sucesso (HASTIE; WOJEWODA, 2015; KARLSEN et al., 2005).

Essa abordagem é conhecida normalmente como triangulo de ferro (ATKINSON, 1999) foi amplamente utilizada pela empresas na década de 1980 (MORRIS; PINTO; SÖDERLUND, 2011), sendo inclusive a o critério de sucesso usado pelo Chaos Report até hoje (HASTIE; WOJEWODA, 2015).

Apesar do triangulo de ferro de amplamente usado como medida de sucesso, Baccarini (1999) e Karlsen et al.(2005) colocam que dois aspectos devem ser considerados ao determinar um sucesso de um projeto:

- O sucesso do gerenciamento do projeto, geralmente medido por critérios relacionados à gestão do projeto, focado em aspectos ligados ao processo, como ele foi conduzido, e realização bem-sucedida do tempo, custo e objetivos de qualidade.

- O sucesso do produto, geralmente mensurados por critérios de usuário e efeito, o qual trata de efeitos relacionados ao produto final, geralmente medidos após o termino do projeto.

\subsection{Metodologia de gestão de projetos da SISP}

Segundo Araújo, Pinheiro Júnior e Abreu (2016), diversas áreas do governo federal vêm implantando o modelo de gestão de projeto na busca de entrega de seus resultados de forma eficaz, eficiente e efetiva. Um desses esforços foi o desprendido pelo Sistema de Administração dos Recursos de Tecnologia da Informação (SISP), através da elaboração da sua Metodologia de Gerenciamento de Projetos MGP-SISP, focada nas melhores prática do Guia de Gerenciamento de Projetos (Guia PMBOK) e na vivência de diversos órgãos públicos que compõem o SISP, considerando legislação que rege o processo licitatório e a Instrução Normativa SLTI/MP no 04 de 2010 (BRASIL, 2011, 2014).

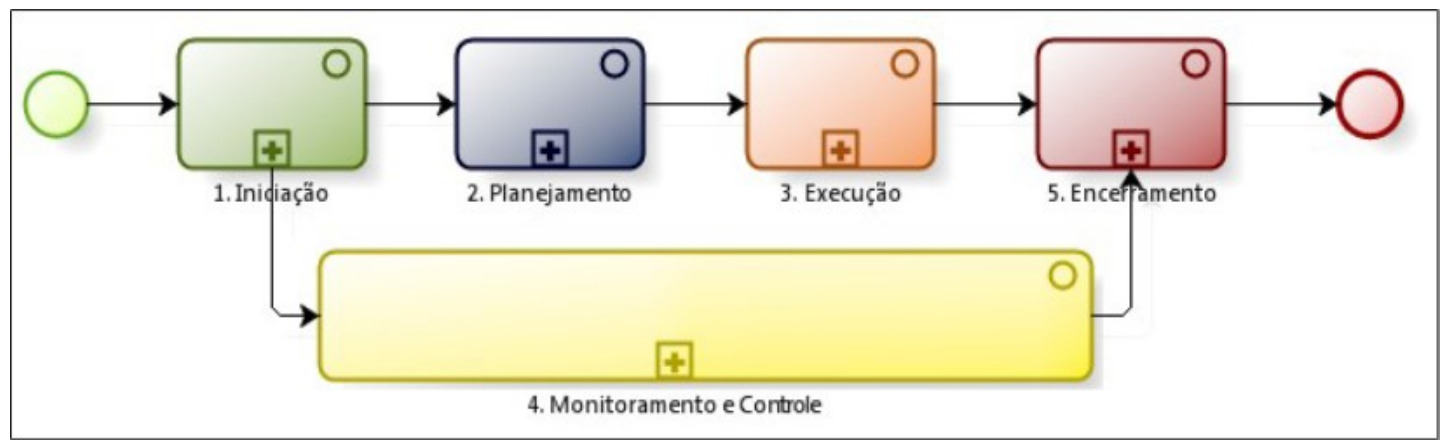

Figura 1: MGP-SISP.

Fonte: produzida pelo autor. 
Uma Proposta de Gestão de Projetos Para uma Universidade Pública Revista Gestão.Org, v. 16, Edição Especial, 2018. p. 264-278

ISSN 1679-1827

http://www.revista.ufpe.br/gestaoorg

O MGP-SISP foi construído a partir da composição de 20 processos distribuídos nos 5 grupos de processos descritos pelo PMI, iniciação, planejamento, execução, monitoramento e controle e encerramento(BRASIL, 2011), conforme descrito na Figura 1.

Em cada um desses grupos, os processos são identificados com numa numeração em sub níveis de seu respectivo grupo, como por exemplo o processo "1.2. Elaborar Termo de Abertura do Projeto" que faz parte do grupo de processo de iniciação (que tem a numeração 1). Esses processos são detalhados a partir da descrição de seus objetivos, entradas, atividades e saídas.

\subsection{Nivel de Gerenciamento de Projetos de TI segundo TCU}

O Administração Pública Federal (APF), frente a necessidade de adotar práticas de governança previstas em leis, regulamentos, normas técnicas e modelos internacionais de boas práticas, vem realizando desde 2010, através da Secretaria de Fiscalização de Tecnologias da Informação (SEFTI) do Tribunal de Contas da União (TCU) um levantamento de governança de ti denominado índice de governança de TI (iGovTI) (BRASIL. TCU, 2014a).

No contexto institucional, o gerenciamento de projetos de TI segundo o, tem impacto direto no sucesso nas ações planejadas a nível da organizacional pública, considerando elas são muitas vezes suportadas por recursos de TI, como sistemas de informação e soluções de infraestrutura de tecnologia, dessa forma, o fracasso dos projetos de TI geralmente compromete os resultados de ações institucionais (BRASIL. TCU, 2014c).

Cada um dos itens avaliados pode assumir os valores descrito a quadro seguinte, de acordo com a resposta escolhida. Esse valor, é multiplicado pelo peso de cada resposta, e computa o resultado final do nível avaliado.

\begin{tabular}{|l|l|c|}
\hline \multicolumn{2}{|l|}{ Categoria de Resposta } & \multicolumn{1}{c|}{ Descrição da Categoria } \\
\hline Não adota a prática & $\begin{array}{l}\text { A organização ainda não adota a prática, bem como não iniciou } \\
\text { planejamento para adotá-la. }\end{array}$ & 0,0 \\
\hline $\begin{array}{l}\text { Iniciou ou concluiu } \\
\text { plano para dotar a } \\
\text { prática }\end{array}$ & $\begin{array}{l}\text { A organização ainda não adota a prática, mas iniciou ou concluiu } \\
\text { planejamento visando adotá-la, o que se evidencia por meio de } \\
\text { documentos formais (planos, atas de reunião, estudos preliminares etc.). }\end{array}$ & 0,2 \\
\hline $\begin{array}{l}\text { Adota parcialmente a } \\
\text { prática }\end{array}$ & $\begin{array}{l}\text { A organização iniciou a adoção da prática, que ainda não está } \\
\text { completamente implementada, conforme planejamento realizado; ou a } \\
\text { prática não é executada uniformemente em toda a organização. } \\
\text { Há, pelo menos, uma instância de execução da prática e os artefatos } \\
\text { produzidos são evidências dessa execução. }\end{array}$ & 0,5 \\
\hline $\begin{array}{l}\text { Adota integralmente a } \\
\text { prática }\end{array}$ & $\begin{array}{l}\text { A organização adota integralmente a prática apresentada, de modo } \\
\text { uniforme, o que se evidencia em documentação específica ou por meio } \\
\text { do(s) produto(s) ou artefato(s) resultante(s) de sua execução. }\end{array}$ & 1,0 \\
\hline
\end{tabular}

Quadro 1: Valores atribuídos por categoria de resposta do questionário do iGovTI

Fonte: Adaptado do Relatório de Governança de TI(Brasil. TCU, 2014c) e Questionário de Governança de TI (Brasil. TCU, 2014a)

Uma vez aplicado o questionário de método de calculo, as organizações podem classificar sua capacidade de governança, a partir de quatro estágio de capacidade: o inicial: iGovTI menor que 0,3 ; o básico: iGovTI maior ou igual a 0,3 e menor que 0,5 ; o intermediário: iGovTI maior ou igual a 0,5 e menor que 0,7 ; e o aprimorado: iGovTI maior ou igual a 0,7 (BRASIL. TCU, 2014c).

Apesar dessa nota ser aplicada à organização como um todo, a mesma pode ser atribuída por dimensão ou desdobrada por questão (BRASIL. TCU, 2014c), dessa forma, essa categorização pode ser empregada nas questões 
relacionadas a gestão de projetos, e serve como base para avaliação do nível de gestão de projetos de TI em instituições públicas.

Dentre as conclusões tomadas pelo Tribunal de Contas da União em seu relatório (BRASIL. TCU, 2014b), pontua-se a alto percentual de instituições sem prática de gestão de projetos instituída ( $42 \%$ iniciaram o plano ou não adotam) e também o alto percentual de organizações que não possuem um escritório de projetos (59\% iniciaram plano ou não adotam), o que aumenta o risco de insucesso de projetos, principalmente se considerado a alta complexidade de projetos de TI, o que pode acarretar em baixo resultados para as instituições avaliadas (BRASIL. TCU, 2014b).

\section{Proposta de Metodologia de Gestão de Projetos}

A presente proposta foi definida e implementada na Diretoria de Tecnologia da informação (DTI) da Universidade Federal do Cariri (UFCA), bem como ferramentas utilizadas e desenvolvidas para dar apoio a sua implantação, de forma a corroborar com os objetivos, sendo composta por:

- Uma estrutura de projetos, focada em entregas, nos moldes clássicos de estrutura analítica de projetos;

- Um Processo de Gestão adequado ao nível inicial de gestão de projetos, de forma a possibilitar a formalização e acompanhamento dos projetos;

- Aplicação e adaptação de ferramenta de gestão de projetos que dê suporte a execução e acompanhamento das atividades relacionadas ao projeto;

- Um painel para acompanhamento e notificação de informações relacionadas aos projetos, aberta a consulta pública.

\subsection{Metodologia Utilizada}

Para implementar o gerenciamento de projetos de tecnologia da informação na UFCA, foi necessário seguir 2 etapas: a de definição e a etapa de implementação.

$\mathrm{Na}$ etapa de definição, o foco foi em definir quais práticas e ferramentas de gestão de projetos eram possíveis de ser implementadas no nível de maturidade o qual a universidade se encontrava, dessa forma, foi escolhido como base o MGP-SISP, já que a universidade é órgão integrante da SISP.

Frente a isso, os projetos piloto foram utilizados como teste de quais processos e artefatos poderiam ser implementados no contexto da UFCA, para isso, tomou-se como meta estabelecer processos que permitissem a formalização e o acompanhamento dos projetos. Foram escolhidos 3 projetos piloto, um de cada coordenadoria da DTI, garantindo que o processo fosse aplicável tanto na coordenadoria de sistemas, coordenadoria de infraestrutura, quanto na coordenadoria de governança.

Uma vez definida o modelo de gestão dos projetos, o segundo momento focou em implementar o processo definido em toda universidade, e estabelecer um escritório de projetos para acompanhar o andamento da carteira de projetos e garantir que todos os projetos criados estivessem alinhados com o planejado no Plano Diretor de Tecnologia da Informação.

As seções seguintes descrevem o processo e ferramentas implementadas.

\subsection{A Estrutura dos Projetos}

O PMBoK define EAP como uma decomposição hierárquica do escopo total do trabalho que será executado, e aborda duas formas de decompor: através de produtos de trabalho (abordagem bottom-up), a qual compõe o projeto a partir do trabalho a ser realizado pela equipe; ou através de entregas verificáveis ( abordagem top-down), a qual decompõe o projeto nos resultados que desejam alcançar (PMI, 2017).

Essa segunda estrutura foi escolhida para o contexto da pesquisa, devido a facilidade de acompanhamento dos vários interessados do projeto, e considerando a abordagem para medição do progresso. Importante considerar ainda, que com uma equipe nova e pouco experiente, decompor o projeto no que se espera entregar, com o apoio e validação dos diversos interessados acaba se tornando mais fácil que decompor a partir do trabalho que se espera produzir. A estrutura escolhida é melhor demonstrada na Figura 2.

Consequente da visão do projeto em entregas verificáveis, o progresso do projeto não se dá pela evolução do 
Uma Proposta de Gestão de Projetos Para uma Universidade Pública Revista Gestão.Org, v. 16, Edição Especial, 2018. p. 264-278

ISSN 1679-1827

http://www.revista.ufpe.br/gestaoorg

cronograma e execução do planejamento, e sim, pela evolução do escopo, que na EAP, está representado através das entregas. Isso aproxima a forma clássica de ver o projeto como um conjunto de tarefas a serem feitas, com seu progresso estimado pelo percentual de execução do planejamento(SOMMERVILLE, 2011), para uma abordagem mais próxima de um gerenciamento ágil de projetos (APM - Agile Project Management), onde a medida de progresso se dá pelo valor entregue (FOGGETTI, 2014; KARLESKY; VANDER VOORD, 2008).

Dessa forma, para estimar o progresso do projeto, é utilizado estimativa ágil, geralmente estimada em Story Points (FOGGETTI, 2014) adaptado para uma escala de percentuais do projeto, ao invés da escala tradicional baseada na proporção áurea. Essa escala diferente e mais próxima ao entendimento dos interessados faz-se necessário, remetendo própria medida de progresso, o percentual de conclusão.

O cronograma do projeto, de forma similar a estimativa, é dado em termos das entregas, definindo a data prevista a partir do esforço da entrega e do tempo de conclusão de resultados(entregas similares) em outros projetos.

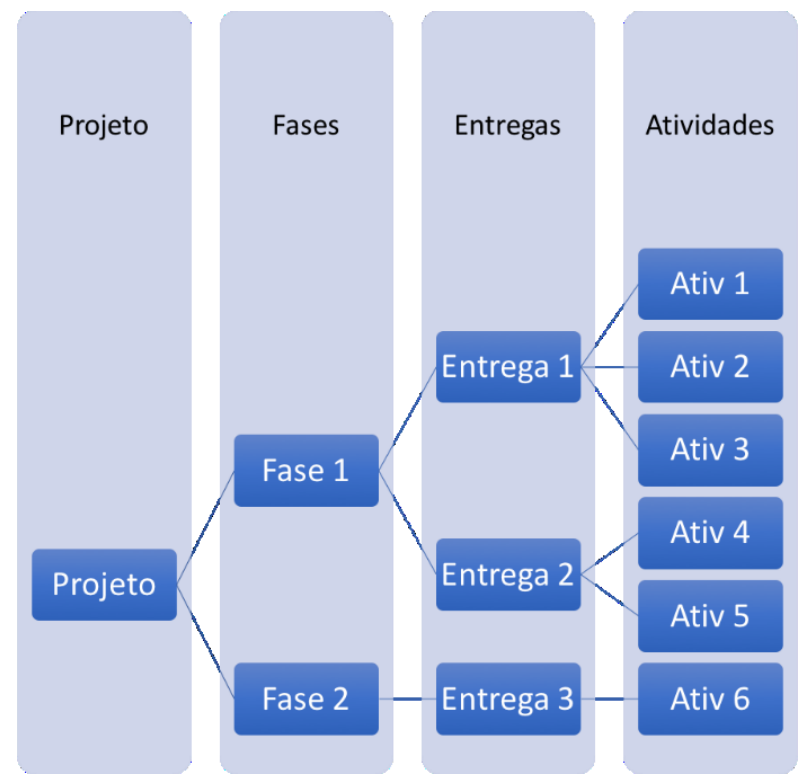

Figura 2: Estrutura Analítica dos Projetos.

Fonte: produzida pelo autor.

\subsection{O processo de gestão de projetos}

O processo definido foca em práticas gerência dos projetos, elaborado a partida da Metodologia de Gestão de Projetos do SISP (MGP-SISP), adaptado a partir da priorização de grupos de processos mais críticos e aplicáveis a um nível inicial de gestão de projetos.

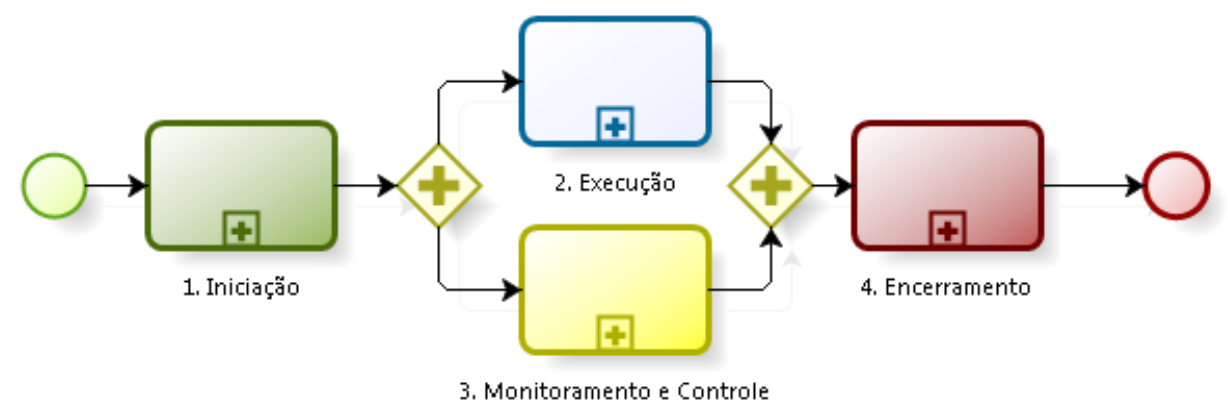

Figura 3: Visão geral do Processo de Gestão de Projetos.

Fonte: produzida pelo autor. 
Os processos do PGP-DTI estão organizados em 4 grupos de processos: Iniciação, Monitoramento e Controle, Execução, e Encerramento, um ciclo de vida simplificado, onde o planejamento é combinado no processo de iniciação (o plano inicial do projeto) e da execução (o replanejamento e ajustes do plano), conforme exibido da Figura 3.

$\mathrm{Na}$ Iniciação, temos processos para definir o planejamento inicial de um novo projeto, por meio da obtenção da autorização formal para início de sua execução. Esse plano inicial deve alinhar o projeto com a ação mapeada do PDTI, identificar objetivos, clientes e equipe do projeto, além da EAP com prazos e pesos estipulados. Esse planejamento revisado pelo escritório do projeto e aprovado pelas partes interessadas. É importante pontuar, que a identificação e autorização inicial dos projetos de TI que são executados em órgão federais, devem ser previamente autorizados por uma entidade deliberativa que representem as áreas fins, o Comitê Gestor de TI.

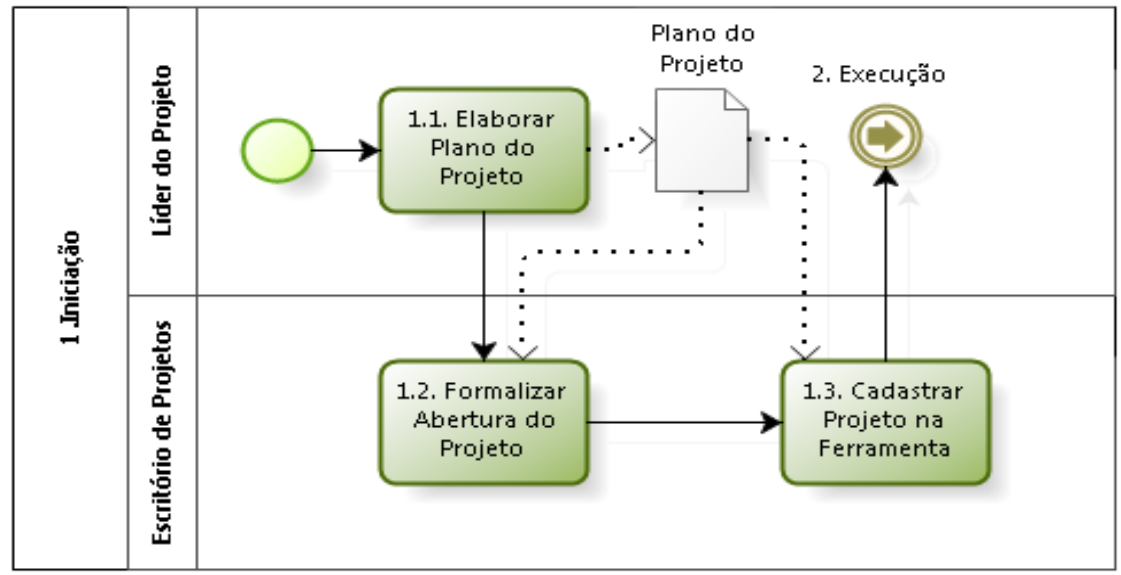

Figura 4: Processos de Iniciação.

Fonte: produzida pelo autor.

Na Execução, apresentam-se os processos relacionados a estimar e gerir as atividades necessárias para realizar as entregas, bem como validar os produtos gerados pelo projeto e obter feedback do trabalho realizado.

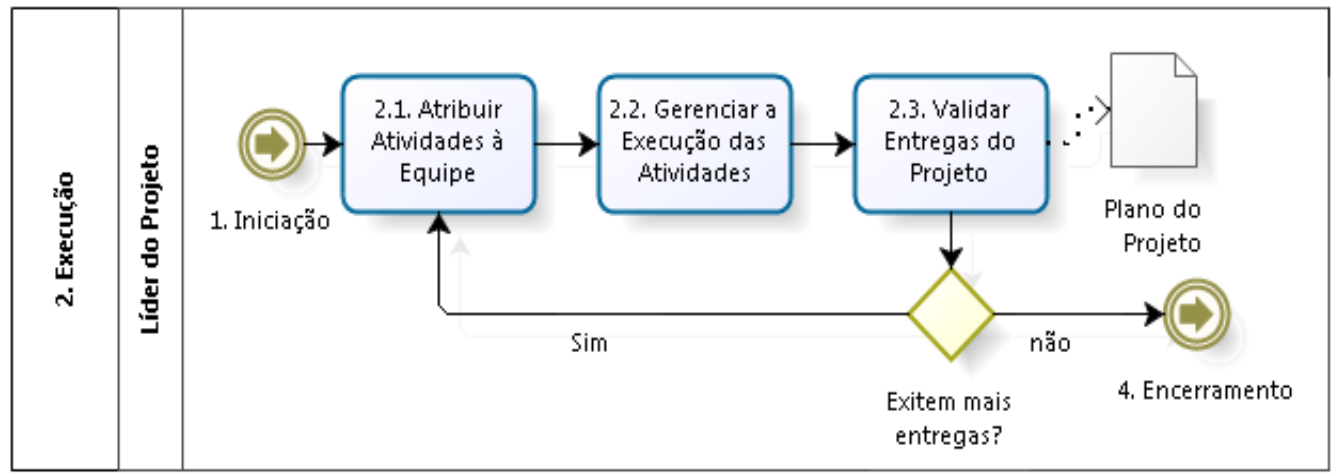

Figura 5: Processos de Execução.

Fonte: produzida pelo autor.

Já na fase de Monitoramento e Controle encontram-se os processos que acompanharão o progresso dos projetos, de forma que possíveis problemas possam ser identificados no momento adequado e que possam ser tomadas ações corretivas e preventivas. 


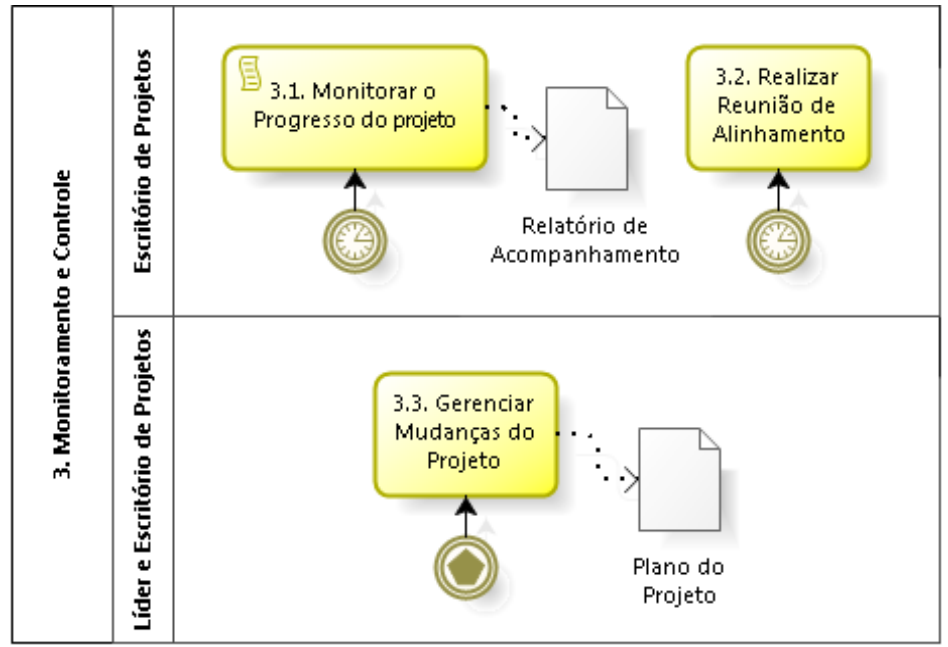

Figura 6: Processos de Monitoramento e Controle.

Fonte: produzida pelo autor.

Por fim, no Encerramento estão os processos para finalizar o projeto, e registar seus resultados, bem como as lições aprendidas durante sua execução.

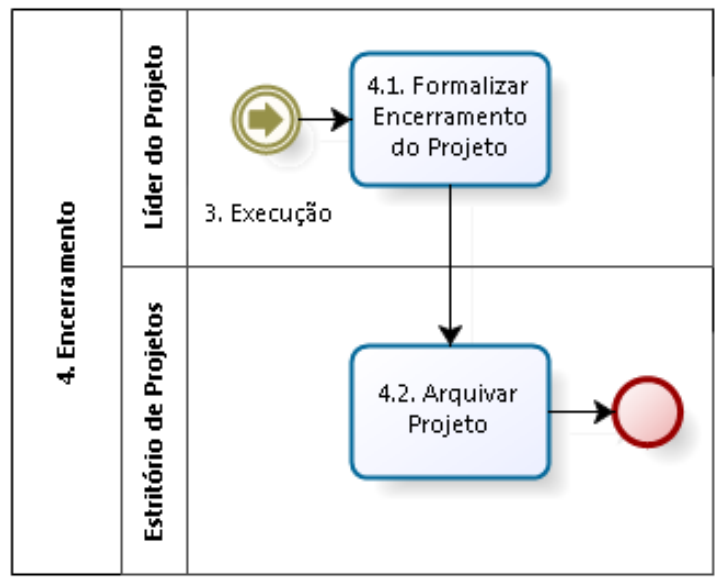

Figura 7. Processos de Encerramento.

Fonte: produzida pelo autor.

\subsection{O software de gestão dos projetos}

A escolha do software utilizado para dar suporte ao gerenciamento dos projetos partiu da necessidade específica do contexto da universidade pública, dessa forma, os pré-requisitos obrigatórios para ferramenta foram: estar sob licença livre; ter suporte a extensões; e disponível em plataforma web. Considerando esse critério, três ferramentas foram selecionadas: o DotProject, Redmine e Web2Project. De forma a facilitar a escolha da ferramenta, outros critérios foram estipulados, sendo esses:

- $\quad$ suporte de idiopas para português;

- login com LDAP, compatível com o sistema gestão de identidade Comunidade Acadêmica Unificada (CAFe);

- experiência da equipe;

- API ReST para integrar com outras ferramentas;

- campos personalizados; 
- $\quad$ e Integração com e-mail, meio formal de comunicação formal da Universidade.

\begin{tabular}{|c|c|c|c|c|c|c|c|}
\hline$\square$ & $\#$ & Tipo & Situação & Título & Atribuído para & Esforço & \% Terminado \\
\hline$\square$ & 5956 & Fase & FAZENDO & Fase 1 & & & \\
\hline$\square$ & 5958 & Entrega & FAZENDO & , Entrega 1 & & 30 & \\
\hline$\square$ & 5961 & Atividade & FEITO & , $\star$ Atividade 1 & Samuel Rodrigues & & \\
\hline$\square$ & 5962 & Atividade & FAZENDO & , $\star$ Atividade 2 & Samuel Rodrigues & & \\
\hline$\square$ & 5959 & Entrega & FAZENDO & , Entrega 2 & & 20 & \\
\hline$\square$ & 5963 & Atividade & FAZENDO & , $\star$ Atividade 3 & Samuel Rodrigues & & \\
\hline$\square$ & 5957 & Fase & A FAZER & Fase 2 & & & \\
\hline ○ & 5960 & Entrega & A FAZER & , Entrega 3 & & 50 & \\
\hline ○ & 5964 & Atividade & A FAZER & , $\star$ Atividade 4 & Samuel Rodrigues & & \\
\hline$\square$ & 5965 & Atividade & A FAZER & , $\star$ Atividade 5 & Samuel Rodrigues & & \\
\hline
\end{tabular}

Figura 8: EAP no Redmine.

Fonte: produzida pelo autor.

A partir dos pontos considerando, o Redmine passou a ser o software mais adequado ao contexto da pesquisa.

Para montar a estrutura da EAP na ferramenta, foram classificados os tipos de atividades como Fases, Entregas e Atividades, e configurado um filtro para exibição de demais campos relevantes. Essa configuração refletiu da exibição do projeto, conforme a Figura 8.

Além da classificação e visualizações das Fases, Entregas e Atividades, alguns campos customizados foram necessários. No Redmine, os campos personalizados podem ser tanto associados aos projetos, quanto às tarefas (fases, entregas e atividades), dessa forma os campos customizado que foram criados estão descritos no Quadro 2.

\begin{tabular}{|c|c|c|}
\hline Campo Customizado & Aplicação & Finalidade \\
\hline Início & Projeto & Registar o início planejado do projeto \\
\hline Fim & Projeto & Registrar o fim planejado do projeto \\
\hline Alinhamento Estratégico & Projeto & Registar o alinhamento estratégico do projeto \\
\hline Objetivo & Projeto & Registrar qual o objetivo final do projeto \\
\hline Situação & Projeto & Registrar o estado do projeto \\
\hline Setor Responsável & Projeto & $\begin{array}{c}\text { Registrar o setor responsável do projeto (considerando } \\
\text { inclusão de outros setores) }\end{array}$ \\
\hline Esforço & Tarefa (Entrega) & $\begin{array}{c}\text { Registrar o esforço (o percentual que a tarefa soma ao } \\
\text { progresso geral do projeto) }\end{array}$ \\
\hline
\end{tabular}

Quadro 2: Campos Personalizados no Redmine.

Fonte: produzido pelo autor.

\subsection{O painel de monitoramento dos projetos}

Em conjunto com o processo e software de gestão de projetos, o painel de monitoramento dos projetos visa dispor informações dos projetos, de forma clara e objetiva, de forma a facilitar a comunicação com as partes interessadas, equipe do projeto e comunidade, objetivando solucionar os problemas associados a comunicação de projetos já identificados por outro pesquisadores (Azman et al., 2013; Crawford \& Helm, 2009; Kwak \& Anbari, 2012)

Considerando que o Redmine é focado no gerenciamento de atividades e entregas do projeto pela equipe do projeto 
Uma Proposta de Gestão de Projetos Para uma Universidade Pública Revista Gestão.Org, v. 16, Edição Especial, 2018. p. 264-278

ISSN 1679-1827

http://www.revista.ufpe.br/gestaoorg

e interessados, o painel de monitoramento de projetos disponibiliza uma visão pública dos projetos, considerando o princípio de transparência da administração pública (MARTINS, 2012), proporcionando uma visão atual dos projetos que o setor de TI está trabalhando, a partir dos dados extraídos e processados a partir da API do Redmine.
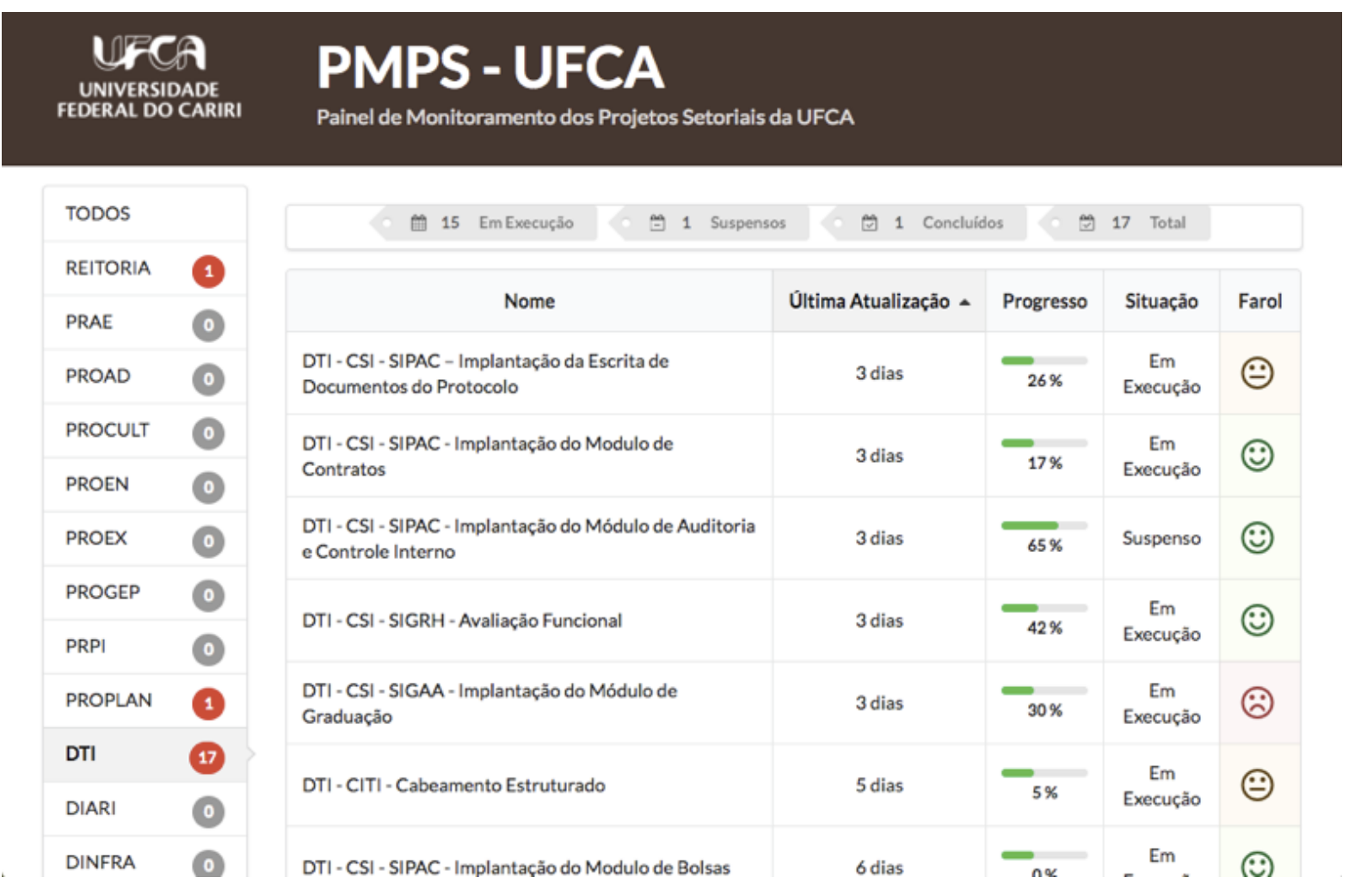

Figura 9: Visão Geral do Painel de Monitoramento dos Projetos.

Fonte: produzido pelo autor.

O painel elaborado pode ser visto nas figuras a seguir, e acompanhado em tempo real em http://paineis.ufca.edu.br/pmps.

A visão de projetos tem o objetivo de ser um resumo dos projetos relacionados a TI, previamente aprovados e formalizados, entretanto, considerando a possível implantação da abordagem de gestão de projetos em outros setores, alguns filtros setoriais foram implementados. Essa visão pode ser vista na Figura 9.

Além da visão geral dos projetos, uma visão de detalhamento de projeto é apresentada através do acesso ao link disponível no nome do projeto, como pode ser visto na Figura 10. Nessa visão são apresentados prazos de início e fim dos projetos, data de ultima atualização, progresso atual do projeto, e avanço do progresso com base no mês anterior, e atraso do projeto quando cabível.

Além disso, um gráfico com o histórico do progresso do projeto ao longo de tempo é exibido, e a lista de entregas do projeto é exibida, com esforço (quando compõe do progresso geral), status da entrega, data prevista, e sinalização quando atrasada ou entregue em atraso. 
Rodrigues, C. S. C.; Soares, F. S. F.

Revista Gestão.Org, v. 16, Edição Especial, 2018. p. 264-278

ISSN 1679-1827

http://www.revista.ufpe.br/gestaoorg

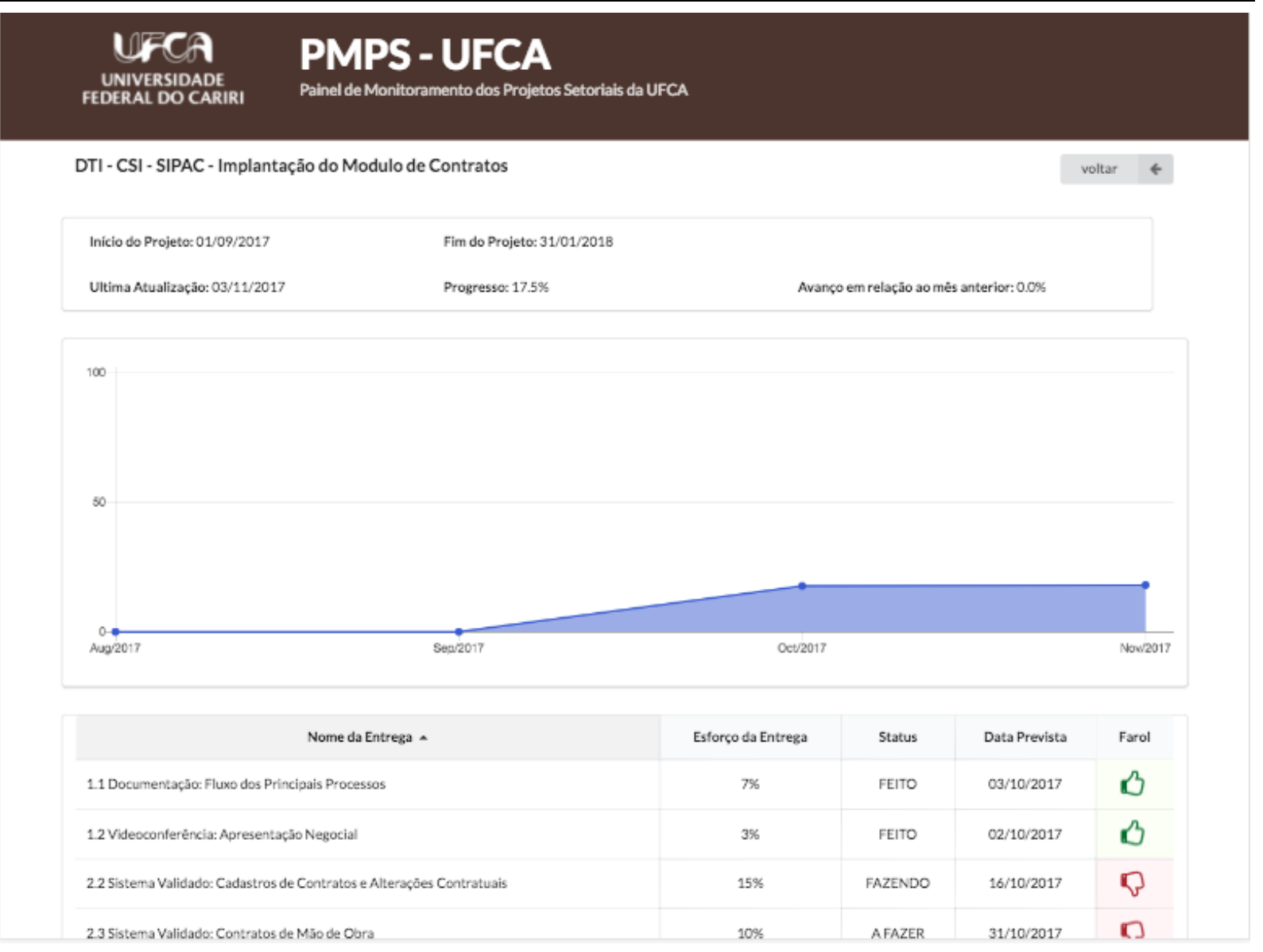

Figura 10: Visão de Detalhamento do Projeto.

Fonte: produzido pelo autor.

\section{Resultados}

Objetivando avaliar o resultado das práticas e ferramentas adotadas pela proposta, o quadro a seguir lista as abordagens utilizadas para avaliar os resultados. O método, resultados e discussão de cada um dos itens descritos acima serão abordados nos subitens seguintes.

\begin{tabular}{|l|l|l|l|}
\hline \multicolumn{2}{|c}{ OBJETIVO } & \multicolumn{1}{c|}{ INSTRUMENTO } & \multicolumn{1}{c|}{$\begin{array}{l}\text { OBJETOS } \\
\text { AVALIADOS }\end{array}$} \\
\hline $\begin{array}{l}\text { Avaliar o gerenciamento } \\
\text { dos projetos segundo o } \\
\text { TCU }\end{array}$ & $\begin{array}{l}\text { Questionário de } \\
\text { autodiagnostico do } \\
\text { IGOVTI disponível no site } \\
\text { do TCU }\end{array}$ & $\begin{array}{l}\text { Gestão dos Projetos } \\
\text { de TI }\end{array}$ & $\begin{array}{l}\text { Gestores do } \\
\text { departamento de gestão } \\
\text { e comitê gestor de ti }\end{array}$ \\
\hline $\begin{array}{l}\text { Avaliar Painel de } \\
\text { Monitoramento }\end{array}$ & $\begin{array}{l}\text { Questionário composto de } \\
\text { perguntas de múltipla } \\
\text { escolha }\end{array}$ & $\begin{array}{l}\text { Painel de } \\
\text { Monitoramento dos } \\
\text { Projetos }\end{array}$ & $\begin{array}{l}\text { Comunidade interna e } \\
\text { externa da Universidade } \\
\text { Federal do Cariri }\end{array}$ \\
\hline
\end{tabular}

Quadro 3: Abordagem de Avaliação da Proposta.

Fonte: produzido pelo autor. 


\subsection{O painel de monitoramento dos projetos}

Objetivando verificar o nível de maturidade do gerenciamento de projetos com base no índice IGovTI, foi aplicada a dimensão 5.6 do questionário de avaliação (Brasil. TCU, 2014b), buscando determinar o nível da instituição, partir da classificação em inicial, básico, intermediário e aprimorado, tomando a nota geral o índice em gerenciamento de projetos. É importante considerar que esse questionário é de auto avaliação.

De forma a entender a evolução dessa nota ao longo da implantação da proposta, o questionário foi utilizado para identificar 3 cenários: O cenário 1, antes da aplicação do processo de gestão de projetos, anterior ao ano de 2016; o cenário 2, durante a aplicação do processo de gestão de projetos, considerando que em 2016 foi completado um ciclo bienal, e foi necessário responder o questionário formalmente para o TCU; e por ultimo o cenário 3, posterior a aplicação da proposta, em 2017.

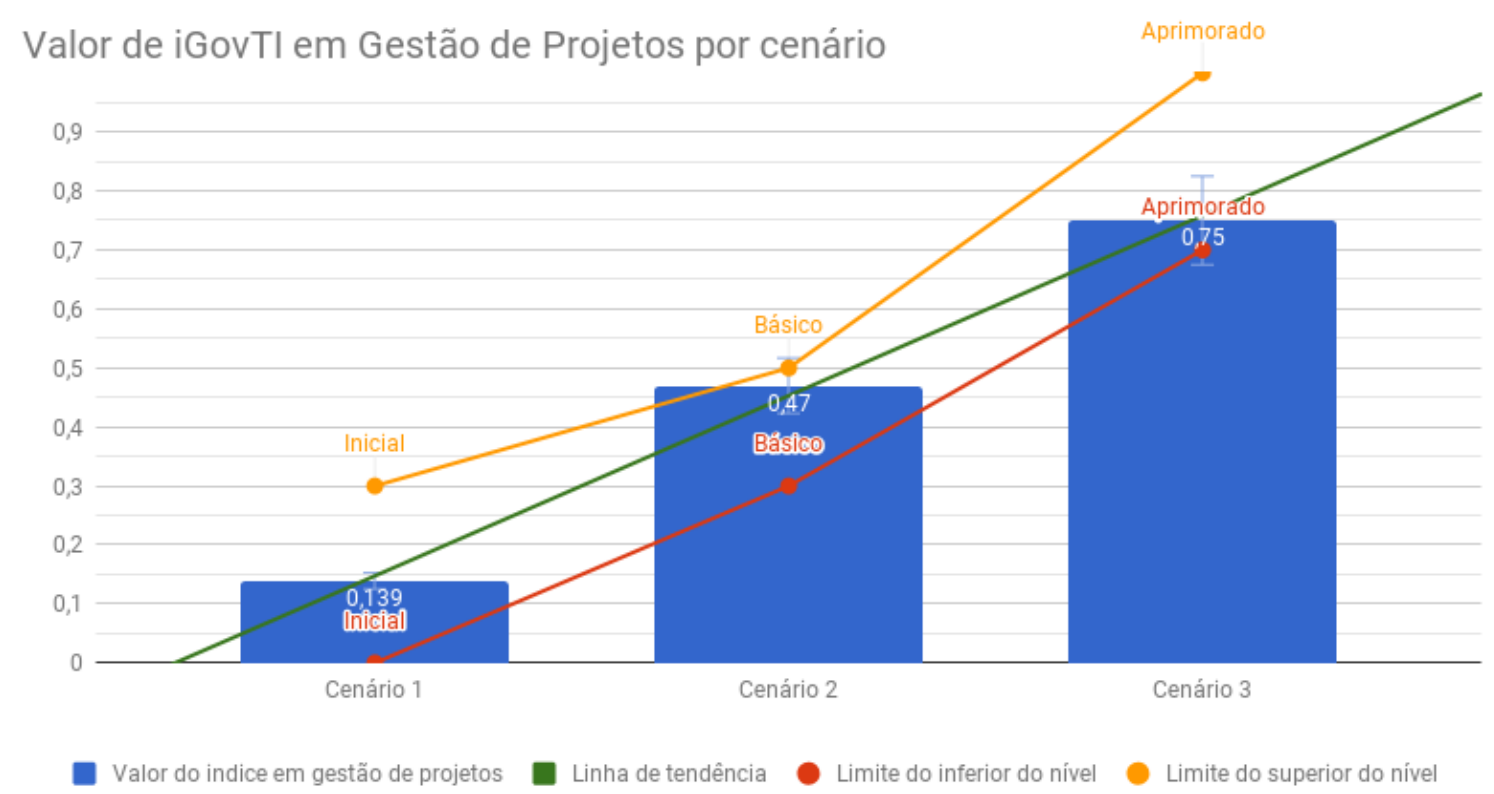

Figura 11: Evolução de IGovTI em Gestão de Projetos na UFCA.

Fonte: produzido pelo autor.

A partir dessas respostas os 3 cenários verificamos a evolução dos projetos de TI da UFCA no índice de gestão de projetos a partir do demonstrado na Figura 11.

Podemos verificar a evolução da UFCA o qual saiu do nível inicial antes da implantação da proposta, atingiu o nível básico durante o ciclo bienal de avaliação, quando a proposta ainda estava em implementação, e ao fim da implementação atingiu o nível aprimorado. Ao consideramos a classificação atual da universidade, verificamos que destoa da maior parte dos órgãos da administração pública, os quais nas perguntas do item Q56, referente a gerenciamento de projetos, apresentam nível inicial (51\%), conforme Levantamento de Governança de TI individual (BRASIL. TCU, 2016).

\subsection{O painel de monitoramento dos projetos}

A avaliação do painel de monitoramento dos projetos tem como principal objetivo verificar o valor da ferramenta e suas informações para comunidade acadêmica, bem como o nível de satisfação. A comunidade acadêmica 
descrita foi identificada a partir do corpo administrativo (composto por servidores efetivos e terceirizados); corpo docente; corpo discente; e comunidade externa.

O formulário para avaliação da ferramenta ficou disponível no próprio painel de monitoramento dos projetos, bem como disseminados nos grupos de discussão da universidade, ficando disponível por período de um mês. As perguntas e respostas aplicadas aos 87 entrevistados pode ser vista na URL https://goo.gl/forms/tGZe7Mtv3jtWAG8A2.

A partir dos resultados apresentados, considera-se que o painel está adequado, atingindo as metas estabelecidas, e comunicando em nível suficiente informações que possibilitem o acompanhamento do projeto por parte da comunidade e dos envolvidos no projeto.

Além disso, considera-se ainda que o painel possibilita maior participação dentro dos projetos desprendidos na Diretoria de TI da UFCA, colaborando com a cultura de gestão de projetos em toda organização.

Os resultados ainda validam aspectos adotados na forma como o projeto é estruturado e estimado, como a decomposição da EAP em entregas que tenham valor para o cliente, e a forma como cada entrega colabora para o avanço no progresso do projeto.

Entretanto, apesar dos resultados positivos, deve-se considerar melhorias alguns pontos, dentre eles, descrição das informações, seu significado, e qual o valor de cada informação no acompanhamento do projeto. É necessário ainda melhorar a forma como as entregas e informações dos projetos são descritas, aproximando ao contexto dos interessados dos projetos, colaborando com o entendimento dessas entregas, do objetivo do projeto, e outras informações associadas.

\section{Conclusões}

Podemos constatar a partir da evolução do índice o iGovTI aplicado ao gerenciamento de projetos, que a instituição pulou do nível inicial ao nível aprimorando na área em questão, atingindo um nível maior que o esperado, e acima da maioria das instituições participantes da avaliação. Isso demonstra o resultado positivo em adotar uma prática simplificada de gestão de projetos, bem como a relevância da presente pesquisa para demais órgão da administração pública.

Apesar de todos os ganhos, é importante considerar, que a UFCA está muito próxima no nível inferior da classificação aprimorada, o que pode facilmente ser diminuído em uma reclassificação das faixas (como ocorreu entre 2012 e 2014), dessa forma, é importante melhorar os resultados gerais, estruturando mais formas de acompanhar, medir e revisar o processo de gestão de projetos.

Outro produto gerado pelo trabalho foi o painel de monitoramento dos projetos, uma ferramenta importante para acompanhamento e proporcionar transparência na execução dos projetos de tecnologia da informação. Tal ferramenta foi avaliada pela comunidade com resultados acima do esperado, cumprindo com o seu papel.

Além dos resultados já descritos, a proposta do presente trabalho corrobora como caso de sucesso para adoção de práticas de gestão de projetos adaptados para o contexto de universidades públicas, bem como para demais setores da UFCA que desejam promover práticas de gestão e transparências dos projetos executados.

\section{Referências}

ARAÚJO, B. D. L. DE; PINHEIRO JÚNIOR, F. M. L.; ABREU, F. P. (2016). Gerenciamento De Aquisições De Soluções De Tecnologia Da Informação Em Uma Universidade Púlica Do Ceará. Revista Gestão Em Anáise, 4(1), 43-53. https://doi.org/10.12662/2359-618xregea.v4i1.p43-53.2015.

ATKINSON, R. (1999). Project management: Cost, time and quality, two best guesses and a. International Journal of Project Management, 17(6), 337.

AZMAN, M. A.; ABDUL-SAMAD, Z.; ISMAIL, S. (2013). The accuracy of preliminary cost estimates in Public Works Department (PWD) of Peninsular Malaysia. International Journal of Project Management, 31(7), 994-1005. https://doi.org/10.1016/j.ijproman.2012.11.008.

BACCARINI, D. (1999). The Logical Framework Method for Defining Project Success. Project Management Journal, Volume 30(Issue 4), 25-32. https://doi.org/10.1086/250095

BRASIL. TCU. (2014b). Levantamento de governança de TI 2014. Brasília. 
Uma Proposta de Gestão de Projetos Para uma Universidade Pública Revista Gestão.Org, v. 16, Edição Especial, 2018. p. 264-278

ISSN 1679-1827

http://www.revista.ufpe.br/gestaoorg

BRASIL. TCU. (2014a). Levantamento de governança de TI 2014. TCU - Tribunal de Contas da União. Brasília. Retrieved from http://portal.tcu.gov.br/lumis/portal/file/fileDownload.jsp?fileId=8A8182A14D78C1F1014D794C4FE4214 0

BRASIL. TCU. (2014c). Relatório de Levantamento da Governança em Tecnologia da Informação. Grupo I Classe V - Plenário. Sessão Plenária (Vol. TC 003.732).

BRASIL. TCU. (2016). Levantamento de governança de TI 2016 - Relatório Individual UFCA, 100.

BRASIL. (2011). Metodologia de Gerenciamento de Projetos do SISP, 1-53.

BRASIL. (2014). Instrução Normativa N 4, De 11 De Setembro De 2014. Dispõe Sobre o Processo de Contratação de Soluções de Tecnologia Da Informação Pelos Órgãos Integrantes Do Sistema de Administração Dos Recursos de Informação e Informática (SISP) Do Poder Executivo Federal. Retrieved from www.governoeletronico.gov.br/sisp-conteudo/nucleo-de-contratacoes-de-ti/modelo-de-contratacoesnormativos-e-documentos-de-referencia/instrucao-normativa-mp-slti-no04

CARVALHO JÚNIOR, M. R. DE. (2012). Gestão de Projetos: da academia à sociedade (1a). Curitiba: InterSaberes.

CLEMENTE, D. H.; MARX, R.; CARVALHO, M. M. DE. (2017). Project management in the public sector: a bibliometric analysis (1988-2014). Revista Gestão Da Produção Operaçães e Sistemas, 12(2), 01-20. https://doi.org/10.15675/gepros.v12i2.1630

CRAWFORD, L. H.; HELM, J. (2009). Government and governance: The value of project management in the public sector. Project Management Journal, 40(1), 73-87. https://doi.org/10.1002/pmj.20107

EDER, S.; CONFORTO, E. C.; SCHNETZLER, J. P.; AMARAL, D. C.; SILVA, S. L. DA. (2012). Estudo das práticas de gerenciamento de projetos voltadas para desenvolvimento de produtos inovadores. Produto \& Produção, 13(1), 148-165. Retrieved from http://seer.ufrgs.br/ProdutoProducao/article/view/24600

FOGGetTI, C. (2014). Gestão Ágil de Projetos. (Casa de Idéias, Ed.) (Pearson Ed). São Paulo: Pearson Education do Brasil.

GRAY, R. J. (2001). Organisational climate and project success. International Journal of Project Management, 19(2), 103-109. https://doi.org/http://dx.doi.org/10.1016/S0263-7863(99)00060-5

HASTIE, S.; WOJEWODA, S. (2015). Standish Group 2015 Chaos Report - Q\&A with Jennifer Lynch. Http://Www.Infoq.Com/Articles/Standish-Chaos-2015, 1-9. $\quad$ Retrieved from http://www.infoq.com/articles/standish-chaos-2015

KARLESKY, M.; VANDER VOORD, M. (2008). Agile Project Management (or, Burning Your Gantt Charts). Embedded Systems Conference Boston (Boston, Massachusetts), (October 2008), 1-16. https://doi.org/10.1145/1101779.1101781

KARLSEN, J. T.; ANDERSEN, J.; BIRKELY, L. S.; ØDEGÅRD, E. (2005). What Characterizes Successful It Projects. International Journal of Information Technology \& Decision Making, 04(04), 525-540. https://doi.org/10.1142/S0219622005001738

KERR, C. (1982). Os usos da universidade: com post scriptum 1972. Edições UFC, Universidade Federal do Ceará.

KWAK, Y. H.; ANBARI, F. T. (2012). History, practices, and future of earned value management in government: Perspectives from NASA. Project Management Journal, 43(1), 77-90. https://doi.org/10.1002/pmj.20272

MACIEL, D.; TEIXEIRA, R. A. C. (2012). Desafios para implantar uma gestão estratégica em organizações públicas. Revista Científica Tecnologus, 7, 8.

MARTINS, H. (2012). Lei da Transparência e sua aplicação na Administração Pública: Valores, direitos e tecnologia em evolução. Superior Tribunal de Justiça. Retrieved from http://www.stj.jus.br/internet_docs/ministros/Discursos/0001182/LEI DA TRANSPARÊNCIA E SUA APLICAÇÃO NA ADMINISTRAÇÃO PÚBLICA VALORES, DIREITO E TECNOLOGIA EM EVOLUÇÃO.pdf

MORRIS, P.; PINTO, J.; SÖDERLUND, J. (2011). The Oxford Handbook of Project Management. The Oxford 
Rodrigues, C. S. C.; Soares, F. S. F.

Revista Gestão.Org, v. 16, Edição Especial, 2018. p. 264-278

ISSN 1679-1827

http://www.revista.ufpe.br/gestaoorg

Handbook of $\quad$ Project $\quad$ Management, $\quad$ (May), $2009-2011$.

https://doi.org/10.1093/oxfordhb/9780199563142.001.0001

PISA, B. J.; OLIVEIRA, A. G. De. (2014). Gestão De Projetos Na Administração Pública : Um Instrumento Para O Planejamento E Desenvolvimento. $1^{\circ}$ Seminário Nacional de Planejamento e Desenvolvimento, 15. https://doi.org/10.1016/j.pubrev.2015.11.004

PMI. (2017). Um Guia do Conhecimento em Gerenciamento de Projetos (Guia PMBOK). Project Management Institute, Inc. (6th ed.). Newtown Square, PA: Project Management Institute, Inc. https://doi.org/19073-3299

SOMMERVILLE, I. (2011). Software Engineering (9th ed.). Peason Prentice Hall. https://doi.org/10.1145/2019136.2019180

SOUZA, E. G. DE; REINHARD, N. (2015). Uma Revisão Bibliográfica dos Fatores Ambientais que Influenciam a Gestão de Projetos de Sistemas de Informação no Setor Público. Revista de Gestão e Projetos, 06(02), 27-41. https://doi.org/10.5585/gep.v6i2.226

VARGAS, R. V. (2005). Gerenciamento de Projetos, estabelecendo diferenciais competitivos. Brasport, 214. https://doi.org/10.1097/FCH.0b013e31822dda9e 